\title{
Gridshells: integrating design with structural performance: formal and informal form finding
}

\author{
Gonçalo Castro Henriques \\ Universidade Federal do Rio de Janeiro, LAMO-Prourb, gch@fau.ufrl.br \\ Juarez Moara Franco \\ Universidade Federal Rural do Rio de Janeiro, COPPE, juarezfranco@ufrrj.br
}

There is a growing interest in the design and construction of flexible gridshell structures. Although gridshells have been around since the 1960s, they require material resources, technical and mathematical knowledge that have limited their application. Digital processes of conception, fabrication and construction offer-renewed possibilities to tackle these difficulties and to deepen the dialogue between form, material and structural performance. This article documents an empirical and theoretical research on these structures that seeks to integrate gradually the formal and informal processes of Form Finding, considering structural behaviour from the early design stage. The research contributes by reflecting on a series of empirical constructions developed and aims to contribute to the collective debate to expand further gridshells possibilities.

Keywords: Gridshell; formal and informal; Kangaroo; Karamba; form finding.

\section{METODOLOGIA, UMA ABORDAGEM INTEGRATIVA}

A história da Arquitetura e da Engenharia de Estruturas é marcada por uma instigante retroalimentação entre teoria e prática. Em cada um destes campos, a pesquisa é habitualmente desenvolvida de maneira autônoma, com seus próprios fundamentos, métodos e objetivos. Procurando aproximar estes dois campos de conhecimento, documentamos aqui um processo híbrido e interativo entre teoria e prática de Arquitetura e Engenharia de Estruturas, cujos ciclos objetivam a proposição e avaliação de soluções, e se retroalimentem com as informações obtidas. Os produtos desta iniciativa dizem respeito tanto à construção da metodologia quanto aos resultados apresentados neste artigo. Assim o projeto não é visto como um momento isolado, mas antes como um conhecimento progressivo onde se somam contributos, estudando os avanços de outros pesquisadores, acrescentando conhecimento, percebendo onde inovar.

A análise estrutural, com as ferramentas digitais, tem-se tornado mais acessível nas primeiras etapas de concepção da forma, em Arquitetura, embora a interpretação crítica das informações dependenda de pré-requisitos teóricos geralmente associados à Engenharia. A metodologia proposta procura integrar a simulação estrutural e intencionalidade estética desde as primeiras etapas de projeto. A pesquisa sobre gridshells foi iniciada com uma parceria do programa BRAGFOST - Brazilian-German Frontiers of Science and Technology, em que o Arquiteto Tobias Wallisser trouxe a referência das gridshell desenvolvidas por Frei Otto para um workshop realizado no Rio de Janeiro. O desafio consistiu no desenvolvimento de uma gridshell em bambu, com o auxílio da ferramenta de análise estrutural Karamba3D@ (plug-in integrado nos no Rhinoceros $\odot$ e Grasshopper@) para avaliar o comportamento mecânico das soluções. Nesta ocasião, a interpretação dos resultados foi viabilizada pela parceria com o professor Felipe Tavares (UFBA/UFPA). Esta pesquisa continuou na Alemanha, onde o estudo procurou analogias entre gridshells e a tipologia das ocas habitações tradicionais dos povos indígenas da floresta amazônica - com a verificação estrutural apoiada pelo pesquisador Thomaz Vieira (Doutorando Detmold). Em ambas as situações, os experimentos com os modelos físicos foram acompanhados por simulações numéricas antes da construção, sendo que as dificuldades incentivaram a inclusão de cálculo estrutural nas etapas iniciais de projeto. O professor Juarez Franco (Coppe / UFRRJ) foi consultor de cálculo estrutural dos resultados do workshop Tropical Gridshell e mais recentemente foi, convidado pelo autor principal deste artigo, para juntos definirem as diretrizes gerais para o desenvolvimento simultâneo da forma e do cálculo estrutural no workshop "Superfícies Mutáveis" (2020). Com base nos resultados do workshop, a pesquisa posterior procurou integrar processos formais e informais que iremos descrever. 0 desenvolvimento incluiu o relaxamento dinâmico da forma (Kangaroo) no desenvolvimento de propostas para gridshells, que foram analisadas e aprimoradas com análise estrutural, para determinar os esforços internos, deslocamentos e carga crítica de flambagem (Karamba3D). Este artigo apresenta os resultados deste último workshop e desenvolvimentos posteriores.

\section{INTEGRANDO MÉTODOS FORMAIS E INFORMAIS}

Utilizamos aqui a designação de métodos informais para nos referir aos métodos não explícitos utilizados para construir com um determinado material, de acordo com uma técnica, aprimorada por tentativa e erro. A tecelagem, é processo ancestral de entrelaçar tecidos, e um exemplo de um método informal, como acontece com outras técnicas e materiais, como a carpintaria e a madeira, a estereotomia e a cantaria, ou a forja e o metal (Semper, 2004). Construir é fazer o melhor proveito das caraterísticas individuais de cada parte e do material, conjugados num todo. A Tesselagem indica à divisão de uma superfície com formas geométricas, sem vazios. A palavra "tessela" designa a pequena peça utilizada pelo 
artesão para preencher uma superfície, com um determinado material, que valora também o sentido compositivo e estético do conjunto. Estes processos que são transmitidos verbalmente, fazem parte do que o Alexander designa como conhecimento inconsciente, ou não formalizado (Alexander, 1963). Em certos problemas computacionais - relacionados com a geometria e a simulação de processos construtivos - existe a necessidade de incorporar informações sobre materiais e técnicas de edificação. Nesses problemas a abstração do conceito de tesselagem é útil para desenvolver a concepção de superfícies e estruturas espaciais. Uma das técnicas computacionais que podem ser integradas no conceito de tesselagem é a discretização de uma superfície tridimensional. Neste caso, para além do sentido matemático estrito, a discretização pode incorporar também o sentido material e compositivo presentes no saber tradicional da construção a que nos referimos. Outro aspeto relevante de tesselagem, é considerar a técnica de fabricação e montagem no projeto. Lienhard et al., 2013 reviram a utilização de elementos estruturais prétensionados (Active bending) desde os processos intuitivos ancestrais de construir, presentes em cada cultura. No séc. XX referem a incorporação dos processos analógicos, do tipo "a forma segue as forças físicas" com Gaudí, Fuller e Otto, que posteriormente, foram ampliados com os processos numéricos computacionais, fundindo a estética com desempenho estrutural.

A Arquitetura está associada a uma procura intensa da forma. Nesta procura, lida com wicked problems, problemas que requerem a tomada de decisões sem existir um processo algorítmico pré-estabelecido (Buchanan, 2006). A natureza destes problemas abarca um universo amplo de possíveis soluções e inclui fatores subjetivos, recorrendo a métodos heurísticos e à experiência empírica de projeto. A literatura em Arquitetura designa form finding num sentido lato, incluindo tanto as situações de busca de forma utilizando algoritmos computacionais explícitos, assim como métodos informais, sem que os métodos produzam realmente a "otimização" da forma. $\mathrm{Na}$ engenharia o termo "otimização" tem um significado matemático mais preciso, associado com outras ferramentas, processos e objetivos. Neste trabalho, adotamos processos formais e informais para diferenciar processos de tomada de decisão pautados por métodos computacionais explícitos, e processo utilizando saberes não codificados - como os saberes implícitos referidos anteriormente. Os processos formais, tem definições computacionais explícitas, o que Alexander associa à prática autoconsciente. Por outro lado, os processos informais, têm origem no teste empírico do artesão, que nem desenha, nem calcula, mas que inclui fatores intuitivos, nem sempre quantificáveis ou computáveis, mas que resultam da evolução ao longo de gerações. Como referido na literatura sobre gridshells (Toussaint, 2007), o projeto deste tipo de estrutura é a arte de juntar as perspetivas arquitetura e engenharia de estruturas, para conseguir uma solução onde convirjam, de maneira elegante os critérios de ambos os campos de conhecimento.

As gridshell exigem a fusão de conhecimento estético e estrutural. Em engenharia de estruturas, o projeto de um sistema estrutural é tradicionalmente organizado em quatro etapas: (i) concepção estrutural, onde se define a forma do sistema, (ii) pré-dimensionamento, onde se definem as dimensões do sistema com base em critérios empíricos, (iii) análise estrutural, onde se calculam os esforços solicitantes e os deslocamentos da estrutura e (iv) dimensionamento, onde se comparam os esforços solicitantes e a capacidade resistente de cada elemento e do sistema como um todo. Nesta distribuição de tarefas, as equipes de arquitetura são tradicionalmente responsáveis pelas etapas de concepção e pré-dimensionamento, enquanto as equipes de engenharia respondem pelas etapas de análise e dimensionamento. Esta organização "em série", permitiu a especialização de tarefas entre arquitetos e engenheiros, que se cristalizou com o advento das Ėcoles Polytechniques e das Ėcoles de Beaux-Arts há mais de duzentos anos, na França. Por outro lado, esta diferença entre ferramentas, discursos e objetivos dificulta o intercâmbio de informação entre as equipes de arquitetura e engenharia. Para haver um processo integrado, que inclua informações sobre os processos e materiais, é necessária uma troca de informações em paralelo entre as duas equipes. Ou seja, através de uma colaboração estreita desde as primeiras etapas de projeto é possível desenvolver soluções convergentes, que atendam simultaneamente aos critérios de arquitetura e engenharia com menos ciclos de iteração e retrabalho.

\section{GRIDSHELLS}

Uma casca reticulada, em malha ou treliçada é essencialmente uma casca cuja estrutura é concentrada em elementos de barra, numa malha relativamente esbelta, quando comparada com a dimensão total da estrutura (Adriaenssens et al., 2014). Na literatura de engenharia de estruturas, o termo "treliça" é um caso particular de estrutura reticulada, onde os elementos de barra não são submetidos à flexão ou força cortante. Este artigo, relata uma experiência didática dirigida a um público relativamente leigo, em que utiliza os termos casca "treliçada", "em malha", ou "reticulada" de maneira equivalentes. A casca reticulada tensionada é uma superfície estrutural curva constituída por elementos de barra flexíveis. Estes elementos são combinados em uma malha plana que é encurvada durante a montagem. Este encurvamento introduz tensões de flexão na casca reticulada, que é assim designada por "casca reticulada tensionada". A casca reticulada não-tensionada, por outro lado, é uma superfície estrutural curva e reticulada, em que a forma das barras é obtida no processo de fabricação (ao invés da reticula tensionada, onde a forma é obtida na etapa de montagem) e onde os esforços solicitantes são devidos ao peso próprio ou a ação externa variável.

Para a engenharia de estruturas, um "elemento de barra" apresenta uma dimensão (eixo) muito maior do as outras duas (seção), enquanto o "elemento de casca" apresenta duas dimensões maiores do que a terceira (espessura). As cascas reticuladas, por combinarem estes dois sistemas com caraterísticas distintas, pertencem a uma classe especial de estruturas. Se consideramos que suas ligações são do tipo articulado, obtemos frequentemente sistemas hipostáticos; mesmo quando considerado que as suas ligações são rígidas, obtemos sistemas com rigidez relativamente baixa na direção normal à superfície. Por definição, as estruturas de baixa rigidez quando submetidas a carregamentos unitários, apresentam grande deslocamento, que compromete a sua integridade. As gridshells, como estruturas reticuladas, admitem três 
classes de variáveis: (i) formais, relacionadas com a proporção entre o sistema estrutural e as suas partes, (ii) dimensionais, associadas com a medida da seção transversal das barras, e (iii) topológicas, que dizem respeito à relação de adjacência entre as barras. É habitual a arquitetura responder pelas variáveis formais e topológicas e a engenharia tomar as decisões relativas às variáveis dimensionais. Esta divisão, no entanto, depende do tipo de projeto, do grau de entrosamento entre as equipes e das ferramentas que compartilhem. A integração de ferramentas computacionais de arquitetura (CAD) e de engenharia (CAE) permite que as etapas de concepção, pré-dimensionamento, análise e dimensionamento sejam simultâneas, ou seja, que as equipes trabalhem em paralelo, em uma plataforma computacional comum. Compreender as classes de variáveis, de objetivos e de estratégias do projeto com Gridshells são pré-requisitos fundamentais para desenvolver uma modelagem computacional integrada. Existem diversas estratégias de modelagem paramétrica de estruturas, considerando as variáveis formais, dimensionais e topológicas. Uma vez que a medida de desempenho das soluções tenha sido estabelecida pela equipe de projeto, é possível escolher soluções (i) maximizem o desempenho do sistema e (ii) obedeçam a determinados critérios, também designados como "restrições" do sistema. Para tal, é possível ajustar as variáveis por tentativa-e-erro ou através de métodos computacionais, designados genericamente por "métodos de otimização". Antes dos computadores, o form finding era realizado por procedimentos analógicos, como o método das correntes suspensas adotado por Gaudí e pelos construtores desde o período Medieval. Por outro lado, utilizamos processos informais que não são otimização no sentido estrutural estrito. Esta pesquisa procura compatibilizar processos formais e informais (processos implícitos e explícitos), pelo que não tem o objetivo de conseguir a melhor solução possível, mas antes soluções factíveis em conjunto variado de parâmetros e objetivos, alguns mais difíceis de quantificar como o processo de fabricação digital e montagem.

A pesquisa descrita, está mais próxima de um processo top-down, onde a equipe de arquitetura e de estruturas desenvolve o projeto base da "forma como um todo", aprofundando em paralelo as etapas de montagem e detalhamento construtivo. Num processo bottom-up, a equipe arquitetura-estrutura pode restringir o repertório de elementos construtivos e estruturais base, definindo um constrained generative system, ou seja, um sistema generativo restringindo à priori, por exemplo as possibilidades de fabricação e montagem. Com sistemas integrados CAD-CAE-CAM é possível incluir ambas as estratégias em simultâneo.

\section{CLASSIFICAÇÃO DE TIPOS DE GRIDSHELLS}

Existem diversos critérios para a classificação das estruturas do tipo gridshell, entre os quais se podem citar princípios (i) formais baseados na geometria da superfície e de sua malha, (ii) estruturais, norteados pela rigidez do sistema e de seus elementos constituintes ou (iii) construtivos, fazendo referência a diferentes metodologias de fabricação e montagem. Não temos conhecimentos de nenhum conjunto de princípios de classificação universal, que atenda simultaneamente os critérios associados à utilização das tecnologias CAD-CAE-CAM. Apesar de no ponto de vista estrutural todo sistema apresenta comportamento elástico (dentro de certos limites), apresentando maior ou menor rigidez, do ponto de vista construtivo, é importante distinguir entre os sistemas que são curvados in loco no processo de montagem (sistemas flexíveis ou cascas reticuladas tensionadas) daqueles em que a curvatura é imposta no processo de fabricação (sistemas rígidos ou cascas reticuladas não-tensionadas). Relativamente ao grau de complexidade de uma gridshell, encontramos uma classificação, que a partir de uma análise de mais de 60 exemplos, classifica as gridshells de acordo com a forma, estrutura e material (Charest et al., 2019). Cada uma destas categorias é subdividida desde o tipo mais simples até o mais complexo (Figura.1).

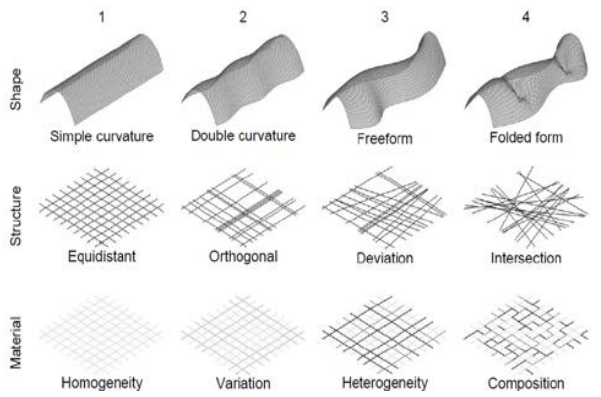

Figura 1: Nível de complexidade das gridshell de acordo com form, estrutura e material, (Charest et al., 2019).

Os autores da pesquisa analisam exemplos desde os anos 60 , identificando ainda dois tipos construtivos, as gridshells elásticas e as gridshells rígidas (elastic and rigid gridshells), designação que no sentido estrutural é questionável, mas quando reportada à construção é importante, para realçar como é obtida a forma final da estrutura. Em engenharia, "elasticidade" é a qualidade do material que permite que ele se deforme sob ação de forças externas e retome sua configuração inicial quando estas forças deixam de atuar sobre o material - ao contrário de um material plástico, que não retorna à sua forma inicial. Adicionalmente, na engenharia, o contrário de rígido é flexível. Por este motivo entendemos ser mais adequado, diferenciar as gridshells "elásticas" como sendo tensionadas e as gridshells "rígidas" como sendo não tensionadas, designação adotada também na descrição que apresentamos sobre gridshells (Adriaenssens et al., 2014).

As gridshells não tensionadas vêm se tornando mais populares com a introdução de métodos de cálculo computacional desde o final dos anos 80 , tendência que é reforçada no final dos anos $90 \mathrm{com}$ a introdução dos processos de fabricação digital. Ainda assim, apesar de que cada processo de obter a forma possa ter as suas vantagens - sendo que os métodos podem ser eventualmente combinados - a construção de gridshells continua a ser complexa, abrindo espaço para pesquisa voltada à simplificação e desenvolvimento de diferentes geometrias e materiais. Para que assim suceda, os autores deste trabalho partem do pressuposto que é fundamental incluir uma análise estrutural, ainda que de baixa resolução, nas fases iniciais de projeto, introduzindo no processo de busca da forma a interação com as propriedades dos materiais e das forças. 


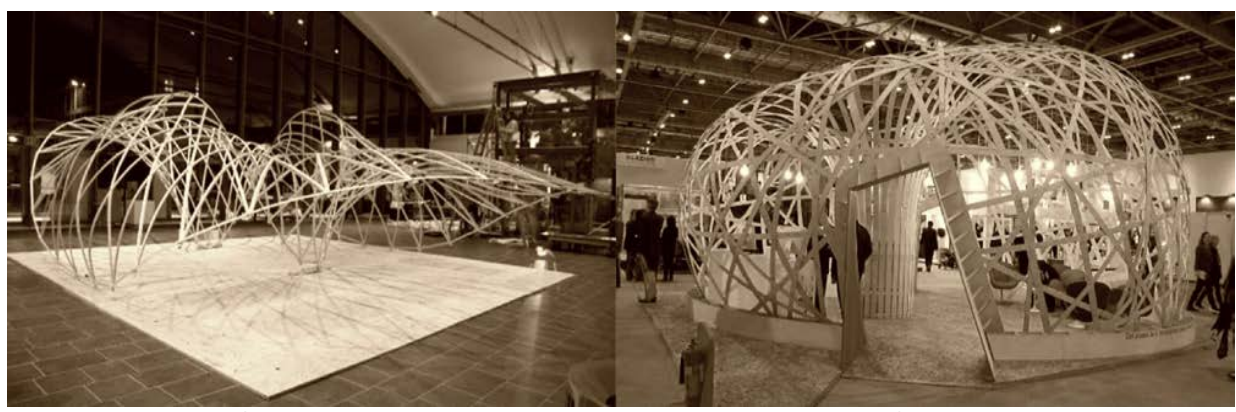

Figura 2: Gridshell tensionadas com ripas de madeira: smart geometry gridshell e Ongreening gridshell.

\section{SÍNTESE EXEMPLOS E TENDÊNCIAS}

Nesta secção apresentamos uma breve síntese sobre exemplos de gridshells construídas, refletindo sobre as suas caraterísticas e atualidade. Embora a invenção das gridshells seja atribuída a Shukhov com a estrutura metálica do Pavilhão Vyksa de 1896, o Multihalle Mannheim de Frei Otto de 1975, é considerado o exemplo "fundador", não só pelas suas caraterísticas espaciais e dimensionais, mas também pelo inovador sistema de malha-dupla de madeira pré-esforçada. Após esta construção, se destaca o EPFL Polydôme de 1991, procurando uma alternativa não tensionada. Nos anos que se seguiram, poucos edifícios foram construídos utilizando a técnica original de Frei Otto, devido às dificuldades de cálculo e do processo construtivo. Shigeru Ban desenvolveu com Frei Otto uma experiência interessante em papel no Pavilhão do Japão em Hannover com em 2000. No desenvolvimento de gridshells tensionadas se destacam o museu Weald and Downland de Happold em 2002 e o Pavilhão Savill Garden, de Howells em 2006. Ambos recorrem a tecnologias recentes para continuar a tradição de Otto de estruturas tensionadas. Continuam, no entanto, a ser soluções complexas, demoradas e dispendiosas. A influência da fabricação digital começa a ser percebida na última década, num conjunto de projetos com gridshells não tensionadas, com madeira lamelada colada de forma pré-definida, como o Centre PompidouMetz e Nine Bridges Country Club de Shigeru Ban em 2008 e 2009, assim com a conhecido Metropol Parasol de Mayer em 2010. Estas experiências, se por um lado simplificaram a montagem em obra, tomam mais complexo o processo de modelagem e fabricação. Nesse sentido, numa fase posterior há um regresso às gridshells tensionadas aprofundando conceitos geométricos e materiais, como por exemplo no pavilhão Solidays' Festival em 2011, que utilizou tubos GRFC (Tayeb et al., 2013), e em outros casos que se utilizaram ripas de madeira, como na Gridshell do Smart geometry de 2012 (Cabrinha, 2014) ou do Almond Pavilion de 2012 (Soriano, 2017). Outra vertente explorada com o relaxamento dinâmico durante a simulação de gridshells tensionadas discretizadas é explorada no ZA Pavilion (Naicu et al., 2014) ou Toledo gridshell 2.0 (D'Amico, 2014) com form-finding digital, havendo ainda exemplos que associam as gridshells com sistemas modulares pré-fabricados com fabricação digital (Mork et al., 2016). Estes pavilhões tensionados introduzem cargas importantes no processo de montagem, sendo as retículas discretizadas com um sistema duplo de barras de madeira. Há exemplos mais recentes da reinterpretação das gridshells tensionadas contínuas na academia com experiências na Universidade Chalmers (Adiels et all, 2017) ou UWE (Harding 2016), em colaboração com o matemático Chris Williams, ou a pesquisa mais recente do The Ongreening Pavilion (Harding et al., 2016). Como exemplos do desenvolvimento gridshells não tensionadas, temos alguns projetos desenvolvidos na academia, com recurso à fabricação digital, utilizando meshes, como por exemplo os projetos Catalyst Hexshell (Kudless, 2012) e o Pavilhão Mesh-gridshell (Naboni, 2016).

Uma questão importante que tem um impacto nas gridshells é a relação entre os materiais e o esquema de construção utilizado (Fernandes et al., 2016). Ainda de acordo com este autor, as gridshells tensionadas podem ser classificadas em 3 tipos, gridshells compressão

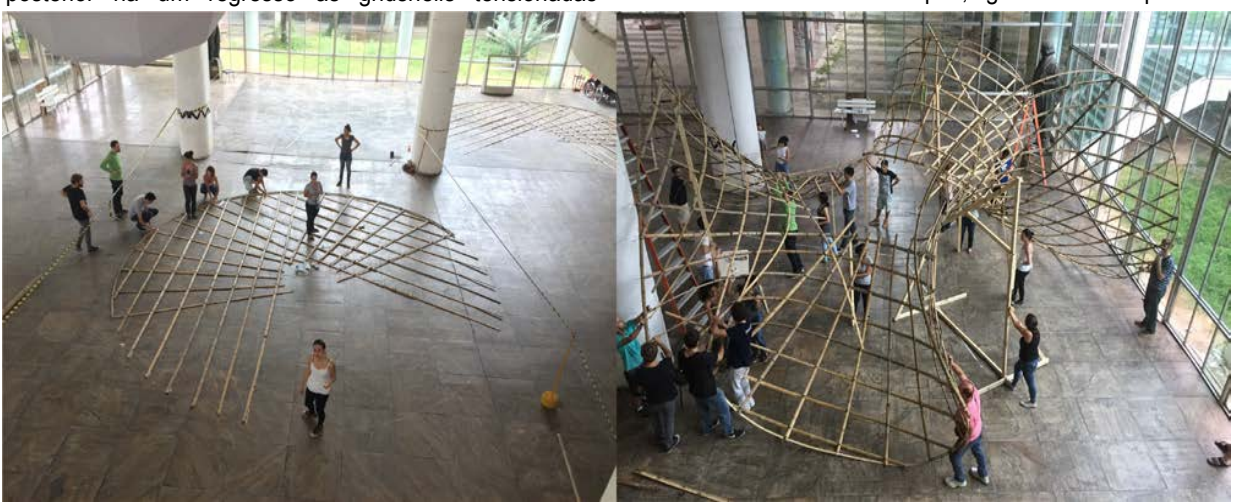

Figura 3: Tropical Gridshell experience, programa BRAGFOST, Brazilian German Frontiers of Science and Technology com apoio Von Humboldt DAAD, coordenado por Gonçalo Castro Henrique e Tobias Wallisser com Daniel Lenz, Março 2018. 
(arcos), tensionadas (catenárias) e mistas, numa classificação simplificada, uma vez que as gridshell podem apresentar uma parte dos elementos de barra sob tração, e outra sob compressão. Refere também que a construção pode utilizar os métodos pull-up (içar para cima, com guindaste), push-up (empurrar desde baixo, com atuadores por exemplo), ease-down (Suspensão desde cima, para adquirir a forma) e o método inflate (Enchimento de Inflável). Há uma tese de doutoramento recente que desenvolveu uma gridshell tensionada pneumática (Quinn \& Gengnagel, 2014). Essa pesquisa estudou os processos de montagem, assim como os processos de form-finding. Ainda de acordo com esta tese, existem outros modelos de form-finding para gridshells, como os modelos de correntes suspensas, definições matemáticas (rede Chebyshev), o método do compasso, entre outros. Finalmente, o trabalho de Quinn \& Gengnagel (2014) resumiu a implementação da construção, dos processos de montagem e dos detalhes de encaixes móveis aplicados em de alguns pavilhões de referência. Estas pesquisas destacam a especificidade dos métodos de form finding em gridshells tensionadas.

Para aprofundar a experiência do Pavilhão Tropical Gridshell Experience, na UFRJ, em 2018 (Wallisser et al., 2019), foi necessário procurar uma dialética entre teoria e prática. Esta dialética aprofundou o estudo de materiais e de processos e possíveis soluções construtivas. Ficamos surpreendidos por encontrar um autor que desenvolveu um método alternativo para conseguir construir gridshells com base em superfícies de Enneper - como a superfície da Tropical Gridshell Experience que contruímos anteriormente. Este autor argumenta que não é possível obter formas como a que obtivemos, recorrendo ao processo de montagem das gridshells tensionadas, o que mostramos que não é necessariamente verdade. 0 referido autor propôs um sistema híbrido para combinar as vantagens das gridshell tensionadas e rígidas para superar estes desafios geométricos (Yuan et al., 2018).

\section{PESQUISA DURANTE WORKSHOP}

O Seminário / Workshop "Superfícies Mutáveis", foi organizado em conjunto pelas Universidades FAU-UFRJ (Brasil), Bio-Bio (Chile), FADU-UNL (Argentina) e UCR (Costa Rica), tendo sido desenvolvidos exercícios complementares de estruturas de acordo com a experiência anterior de cada universidade, entre 9 a 16 Fevereiro 2020. Este evento LAMO foi coordenado por Gonçalo Castro Henriques, Andres Passaro, Pedro Engel e Daniel Lenz, que além da equipe do LAMO, teve a colaboração dos Professores convidados Rodrigo Alvarado, Mauro Chiarella, Carolina Vargas, Juarez Franco e teve ainda a participação de tutores externos como
Alexis Salinas, Paula Ochoa, Luciana Gronda, Martín Veizaga, e de participantes dos vários países.

Os autores deste artigo, conduziram um dos temas do workshop tendo proposto um exercício de projeto, incluindo a modelagem associada com a análise de desempenho estrutural. Os tutoriais preparados visaram implementar esta associação entre a modelagem paramétrica e análise estrutural em tempo real, utilizando o Método dos Elementos Finitos (FEM) no Grasshopper, com o plug-in Karamba3D (Preisinger, 2013). Os participantes foram desafiados a desenvolver um processo com retroalimentação, associando as caraterísticas do tipo de superfície modelada - à densidade, direção e dimensão das barras desenvolvendo diferentes grids- comparando $e$ analisando o desempenho estrutural das várias propostas. Para analisar 0 desempenho consideramos os deslocamentos devido ao peso próprio, flambagem (buckling) e a taxa de utilização da estrutura. Todo sistema estrutural, em situações convencionais de projeto, deve oferecer rigidez e resistência suficiente para não se deformar mais do que o aceitável e não colapsar. As normas brasileiras adotam a filosofia do Método dos Estados Limites, que assume um conjunto de verificações para deslocamentos (Estado Limite de Serviço, ELS) e outra para resistência (Estado Limite de último, ELU). O ELS prevê que nenhum ponto da estrutura apresentará deslocamento $\delta$ maior do que um valor de referência $\delta_{a d m}$; o ELU, por sua vez, impõe que nenhum esforço solicitante $\mathrm{S}$ (força normal, força cortante e momentos) seja maior do que a resistência $\mathrm{R}$ dos componentes ou do sistema estrutural como um todo. Define-se taxa de utilização $t_{x}$ em um elemento como a razão $S / R$. Claramente, uma taxa de utilização $t_{x}=1$ significa que as solicitações se igualaram à capacidade resistente, o que coloca a estrutura (na melhor das hipóteses) na iminência do colapso; $t_{x}>1$ assegura que as solicitações ultrapassaram a capacidade resistente e que a estrutura seguramente entrará em colapso; $t_{x}<1$ nos oferece uma presunção razoável de que a estrutura está segura. As normas brasileiras estabelecem coeficientes de segurança para majorar esforços e minorar resistência, mas elas não se aplicam às condições de projeto deste estudo. Para o workshop consideramos o cálculo de maquetes das gridshells em cartão paraná, fabricadas por corte a laser, nas dimensões máximas de $80 \times 40 \times 0.03 \mathrm{~cm}$, na escala $1 / 10$. Como primeira aproximação, e com o propósito de oferecer aos principiantes um primeiro contato com a análise estrutural, tão simples quanto possível, adotamos coeficientes de ponderação genéricos e, provavelmente, muito a favor da segurança. Impusemos que (i) nenhuma estrutura deveria apresentar deslocamento superior a 1/200 do menor vão entre apoios, (ii) a taxa de utilização não deveria ultrapassar $0.90(90 \%$ de taxa de utilização) quando considerados os elementos
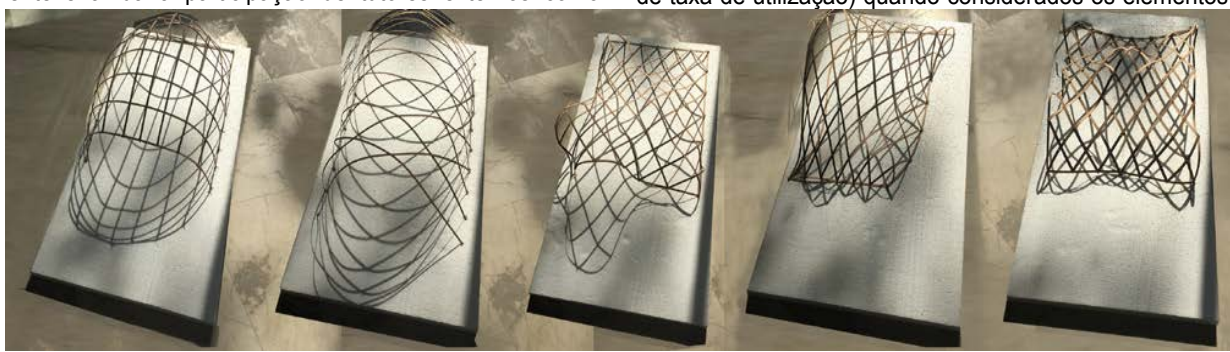

Figura 4: Maquetes físicas gridshells desenvolvidas no workshop superfícies mutáveis, UFRJ 2020. 


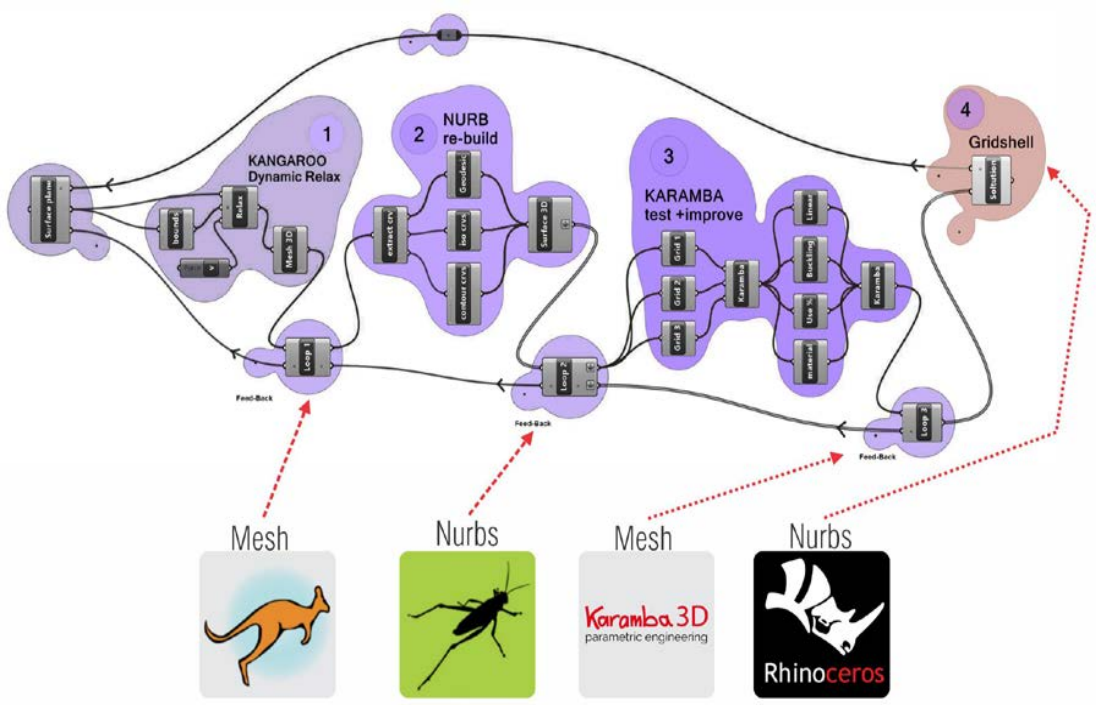

Figura 5: Processo iterativo de projeto, representação simbólica em programação visual com Grasshopper, mas incluindo feed-back:

1-Relaxamento dinâmico (Kangaroo), 2-Mesh to Nurbs, 3-Nurbs to 2d curves e Análise FEM (Karamba) 4- resultado Nurbs

de barra, e adotamos um fator de segurança igual a 3 para o comportamento do sistema como um todo (o fator de carga obtido pela análise de estabilidade elástica maior do que três). Escolhemos o Karamba3D por permitir uma interação expedita, em tempo real, em detrimento de uma análise de elevada precisão, mais demorada e reservada a especialistas. Diferentes pontos de vista sobre as expectativas de desempenho das soluções foram explicitados durante a preparação do algoritmo. Quando consideramos em simultâneo, princípios de Arquitetura e Engenharia, a melhor solução arquitetônica pode não ser a mais resistente ou mais rígida, mas não devem ser violadas as restrições de segurança. Para nortear o processo de procura da forma utilizamos processos formais e informais de form finding, atendendo a exigências técnicas e estéticas, bem como a resultados numéricos que asseguravam desempenho estrutural adequado. As soluções combinaram métodos digitais, com modelos de teste físicos que permitiram testar além da estabilidade, o tipo de ligações e a sequência de montagem.

Os participantes foram introduzidos na programação visual e na análise estrutural no Karamba3D para desenvolverem um projeto de uma gridshell, incluindo, desde o início, o pensamento formal, material e processual. O tipo de problema e a metodologia foram aplicados para a concepção de uma estrutura para um espaço até $6 \times 4$ metros (limitado pelo porte da máquina de corte da laser à escala 1/10). Sugerimos iniciar a modelagem com uma superfície nurbs plana, no solo. Através da manipulação dos pontos de controlo da superfície, os participantes definiram diferentes tipos de superfícies, conforme a posição e número de apoios - por exemplo em todo o perímetro, apoiadas em um, dois, ou três lados e com apoios pontuais. Esta manipulação permitiu definir uma superfície nurbs base contínua, que foi "tesselada" utilizando grelhas regulares, diamante (diagrids) e definidas por curvas geodésicas. Foram desenvolvidas propostas (Figura 4) com a análise FEM, considerando simultaneamente os processos construtivos e materiais a utilizar. O workshop incluiu outros exercícios, desenvolvidas por diferentes tutores, incluindo outros experimentos com form finding em Kangaroo (Piker, 2013), recorrendo a experiências anteriores (Chiarella et al., 2019). Após terminado o workshop, e em tempo de pandemia, O LAMO organizou um grupo para continuar a pesquisa à distância, tendo, no entanto, de passar a utilizar devido à situação apenas processos digitais.

\section{PESQUISA APÓS WORKSHOP}

A pesquisa começou por recolher exemplos construídos, em artigos, livros e teses sobre gridshells que resumimos na seção anterior do artigo. Além dos aspetos referidos foram analisados os processos computacionais de formfinding e de análise de desempenho usados no desenvolvimento dos projetos. Após esta recolha começamos a ver como desenhar o processo de pesquisa para aprofundar soluções. O processo de desenvolvimento utilizado pode ser resumido em 4 momentos: 1 Procura da forma partindo de uma superfície plana e definindo os seus pontos de apoio, com relaxamento dinâmico em Kangaroo; 2 A tradução e redesenho da mesh discretizada para um obter uma superfície NURB contínua, com extração da superfície de diferentes curvas como isocurvas, geodésicas, curvas de contorno, entre outras, para gerar grelhas para gridshells, 3 Avaliação do conjunto de soluções com análise FEM, 4 escolha da superfície nurb final e respetivas curvas. Este processo iterativo está resumido na figura 5 .

O processo de procura da forma utilizou o relaxamento dinâmico no Kangaroo 1, para obter uma solução inicial tipo mesh, que foi trabalhada de acordo com o diagrama acima referido. Os participantes exploraram formas mais complexas que as anteriores, que incluíam descontinuidades topológicas - como um, dois ou três buracos - e testaram mais ativamente os apoios. Neste processo a forma da malha poligonal (poligonal mesh) resultou da interação da malha plana, com forças aplicadas, definindo pontos de apoio, além de propriedades básicas dos materiais. Se utilizarmos 


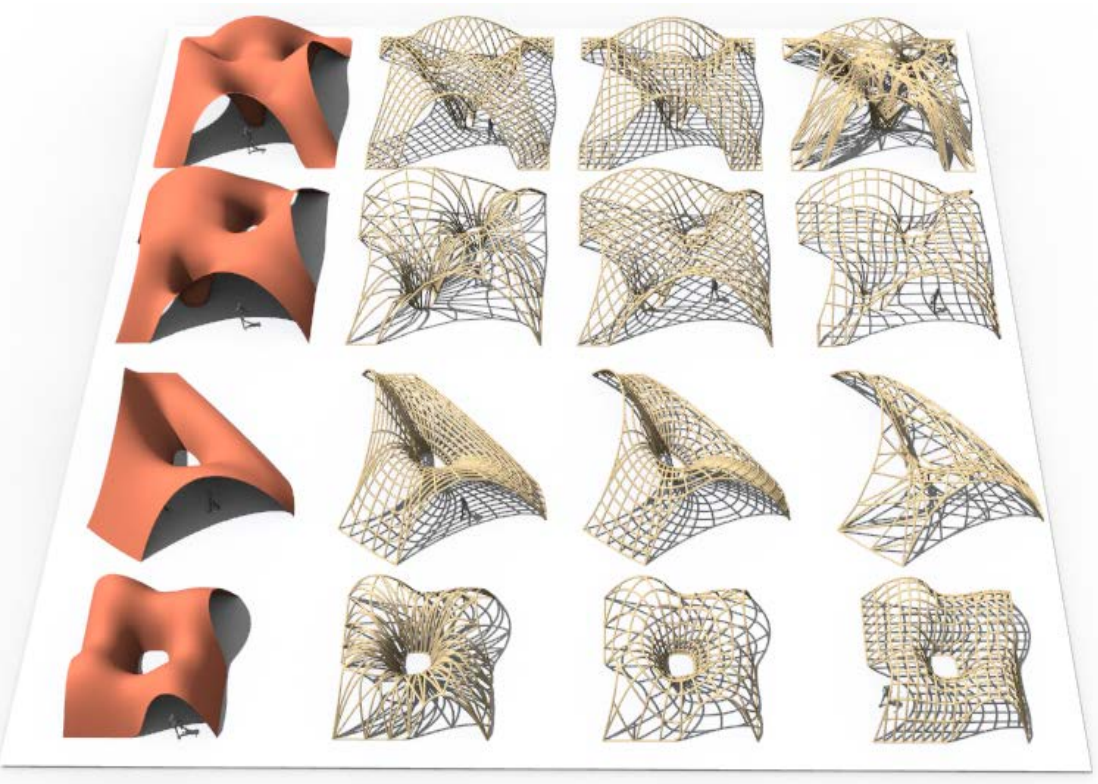

Figura 6: Soluções pesquisa pós-workshop, propostas Maria Eduarda Babo, Vinícius Salles, Ronaldo Lee e Luca Rédua (De cima para baixo), - propostas desenvolvidas pelo grupo, de que resultam vários tipos de grid para cada superfície, testadas no Karamba.

diretamente as malhas poligonais obtidas no kangaroo para construir a estrutura das gridshell, destas utilizam partes separadas (meshes) sem a continuidade topológica das superfícies NURBS. Utilizando diretamente os pontos das malhas, a sua ordem matemática é diferente das nurbs criadas a partir de curvas, o que também dificulta a criação de curvas interpoladas diretamente das meshes, sendo necessário reorganizar as listas de pontos. Então o processo passou por utilizar os pontos para recriar superfícies topologicamente contínuas, o que obrigou a voltar com frequência e modificar a mesh original, ou a alterar os seus apoios, num processo de feedback. Uma vez definida a superfície NURBS foram extraídos vários tipos de curvas como as isocurvas, curvas de contorno (em $z$, $x$ ou y por exemplo), ou geodésicas. Para reconstruir as meshes, especialmente devido às descontinuidades dos buracos, foi importante o trabalho de reordenamento das desenvolvimento assistido, com reuniões semanais em vídeo conferência com o primeiro autor e os alunos Luca Rédua, Maria Eduarda Babo, Ronaldo Lee e Vinícius Salles, do LAMO. Num primeiro momento cada participante desenvolveu 4 superfícies, completando o processo completo de interação descrito. Destas soluções escolhemos em conjunto, uma superfície de cada aluno, para se desenvolveram 4 tipos de grids, resultados que foram sendo discutidos nas reuniões. (Figura 6)

O desenvolvimento da geometria, utilizou processos informais, no sentido da procura empírica de soluções que melhor funcionam em cada geometria proposta pelos pesquisadores, o que requereu sucessivos testes, num processo de feed-back. Neste caso não estamos a falar de otimizar matematicamente, mas a explorar diferentes soluções geométricas e construtivas, que antes de nos
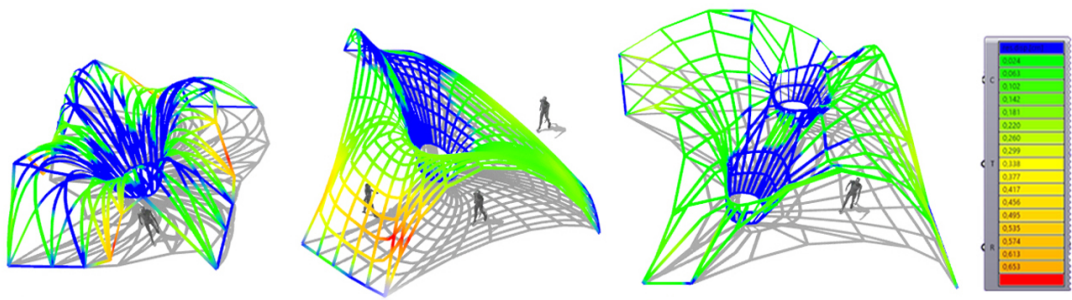

Figura 7: Análise de deslocamentos com Karamba, valores entre 0,021 a 0,065 cm.

listas - a reorganização de meshes segundo critérios matemáticos - mesh sorting methods - é atualmente um amplo campo de pesquisa computacional. Neste momento começamos a avaliar as grids no Karamba3D e a ver as vantagens de cada tipo de grid considerando os processos construtivos e de montagem, servindo-nos das referências recolhidas. Este processo decorreu com o depararmos com esses problemas não podemos antever, nem o problema, nem como resolver. As soluções variam de geometria para geometria, de superfície para superfície. Depois de escolhida cada geometria são testados diferentes parâmetros com o Karamba3D, rejeitando os resultados que estão fora dos valores de referência. Finalmente foi feita uma análise mais 
aprofundada dos resultados estruturais. Na modelagem foram introduzidas as propriedades genéricas da madeira, restringindo a curvatura máxima, e equacionando a relação entre as geometrias desenvolvidas, a sua fabricação e o processo de montagem. Estamos a desenvolver os processos construtivos, com modelos físicos, sendo que pretendemos selecionar as estruturas mais interessantes e factíveis, desenvolvendo o processo construtivo para a construir na escala real. Introduzimos recentemente processos de otimização dos parâmetros com análise multicriterial utilizando o Octopus.

\section{RESULTADOS}

Este artigo, resume os resultados de um estudo progressivo empírico-teórico, mas aplicado de gridshells, em que se procura integrar os processos de formais e informais, para desenvolver estruturas arquitetônicas, procurando introduzir inovação não só na geometria, como no processo. Os autores agradecem a todos os participantes nos diversos experimentos, lembrando que a construção é um ato de superação coletiva. Agradecem, em particular aos criadores do Karamba3D, pelas licenças para uso durante o workshop.

\section{AGRADECIMENTOS}

Aos participaram nos workshops e na pesquisa, em especial nos Seminários / workshops 1 - CURVAS GEODÉSICAS, Geração e Construção, coordenação Andres Passaro, com Clarice Rohde e Nicolle Prado, Nov 2016. 2- TROPICAL GRIDSHELL EXPERIENCE. FAUUFRJ, 5 a 12 Mar 2018. Coordenação: Gonçalo Castro Henriques (FAU-UFRJ / LAMO) e Tobias Wallisser (ABK Stuttgart/ LAVA) com Daniel Lenz, Felipe Tavares. Apoio: BRAGFOST Programa Connect, Fundação Alexander Von Humboldt, DAAD, UFRJ, ABK Stuttgart/LAVA, FAPERJ e CNPq. 3- OCA Traditional Brazilian Construction Reinvented, ABK Stuttgart, Out 2018. Coordenação: Tobias Wallisser (ABK Stuttgart/ LAVA) e Gonçalo Castro Henriques (FAU-UFRJ / LAMO), com: Daniel Lenz, Sebastian Schott e Arnold Walz (Design to Production). 4SUPERFÍCIES MUTÁVEIS, 9 a 17 Fev 2020. Parceria UFRJ, LAMO-PROURB, BIO-BIO (Chile), FADU-UNL (Argentina) e UCR (Costa Rica). Coordenação: Gonçalo Castro Henriques, Andres Passaro, Pedro Engel e Daniel Lenz, LAMO-PROURB. Parceiros convidados: Rodrigo Garcia Alvarado e Mauro Chiarella, com Alexis Salinas, Paula Ulloa (Bio-Bio), Carla Tortul (FADU-UNL), Juarez Franco (COPPE/UFRRJ), Carolina Vargas (UCR).

\section{REFERÊNCIAS}

Adriaenssens, S., Block, P., Veenendaal, D., \& Williams, C. (2014). Shell structures for architecture: Form finding and optimization. In Shell Structures for Architecture: Form Finding and Optimization. Routledge.

Alexander, C. (1963). Notes on Synthesis of Form. In Vasa ( $7^{\text {th }}$ ed.). Harvard, University Press.

Buchanan, R. (2006). Wicked Problems in Design Thinking Design Issues, 8, 5-21. https://doi.org/10.2307/1511637

Cabrinha, M. N. (2014). Lattice shell methodologies. In N. Gu, S. Watanabe, H. Erhan, M. Hank Haeusler, W. Huang, \& R. Sosa (Eds.), Proceedings 19th CAADRIA Conference Rethinking Comprehensive Design: Speculative Counterculture (pp. 191200). CAADRIA.

Charest, P., Potvin, A., Demers, C. M. H., \& Ménard, S. (2019). Assessing the complexity of timber gridshells in architecture through shape, structure, and material classification BioResources, 14(1).

Chiarella, M., Gronda, L., \& Veizaga, M. (2019). RILAB architectural envelopes: From spatial representation (generative algorithm) to geometric physical optimization (scientific modeling). In J. P. Sousa, G. C. Henriques, \& J. P. Xavier (Eds.), - Proceedings 37th eCAADe and 23rd SIGraDi Conference (Vol. 3, pp. 17-24)., Blucher

D'Amico, B., Kermani, A., \& Zhang, H. (2014). Form finding and structural analysis of actively bent timber grid shells. Engineering Structures, Elsevier, 81, 195-207.

Fernandes, J. G., Kierkegaard, P. H., \& Branco, J. M. (2016). Tectonic Design of Elastic Timber Gridshells. In $\mathrm{J}$ Eberhardsteiner, W. Winter, A. Fadai, \& M. Pöll (Eds.), Conference Timber Engineering (pp. 3155-3164). Verlag.

Harding, J., Pearson, W., Lewis, H., \& Melville, S. (2015). The Ongreening Pavilion. In Advances in Architectural Geometry 2014 (pp. 295-308). Springer International Publishing.

Lienhard, J., Alpermann, H., Gengnagel, C., \& Knippers, J. (2013) Active bending, a review on structures where bending is used as a self-formation process. International Journal of Space Structures, 28(3-4), 187-196.

Mork, J. H., Dyvik, S. H., Manum, B., Rønnquist, A., \& Labonnote, N. (2016). Introducing the segment lath - A simplified modular timber gridshell. In W. Winter, J. Eberhardsteiner, \& F.-H. Jourda (Eds.), WCTE 2016 - World Conference on Timber Engineering (pp. 1-8). Verlag.

Naboni, R. (2016). Form-finding to fabrication of super-thin anisotropic gridshell. In R. Martin \& A. Granero (Eds.), Proceedings XX SIGraDi (pp. 418-425). SIGraDi, Blucher.

Naicu, D. (2012). Geometry and Performance of Timber Gridshells (MPhil Thesis) [University of Bath]

Naicu, D., Harris, R., \& Williams, C. (2014). Timber gridshells: Design methods and their application to a temporary pavilion. WCTE 2014 - World Conference on Timber Engineering, Proceedings, 1-9.

Piker, D. (2013). Kangaroo: Form finding with computational physics. In Architectural Design (Vol. 83, Issue 2, pp. 136137). John Wiley \& Sons, Ltd.

Preisinger, C. (2013). Linking structure and parametric geometry. Architectural Design, 83(2), 110-113.

Quinn, G., \& Gengnagel, C. (2014). A review of elastic grid shells, their erection methods and the potential use of pneumatic formwork. In Temmerman \& Brebbia (Eds.), WIT Transactions on the Built Environment (Vol. 136, pp. 129-143). WIT Press.

Semper, G. (2004). Style in the Technical and Tectonic Arts; or, Practical Aesthetics (H. Mallgrave \& M. Robinson (eds.)). Getty Research Institute.

Soriano, E. (2017). Low-tech geodesic gridshell: Almond pavilion. ArchiDOCT, 4(2), 29-38.

Tayeb, F., Caron, J. F., Baverel, O., \& Du Peloux, L. (2013). Stability and robustness of a 300-m2 composite gridshell structure. Construction and Building Materials, 49, 926-938.

Toussaint, M. H. (2007). A Design Tool for Timber Gridshells, The Development of a Grid Generation Tool, MSc Thesis. Delft University of Technology

Wallisser, T., Henriques, G. C., Ribeiro, A., \& Menna, R. L. (2019). Weaving physical-digital networks: Brazil-Germany Integration experience. In S. José Pedro, H. Goncalo Castro, \& X. João Pedro (Eds.), - Proceedings 37th eCAADe 23rd SIGraDi Conference (Vol. 1, pp. 315-324).

Yuan, P. F., Chai, H., \& Jin, J. (2018). Digital form-finding and fabrication of strained gridshells with complex geometries. In Weiguo Xu (Ed.), 23rd International Conference ComputerAided Architectural Design Research in Asia: Learning, Prototyping and Adapting (Vol. 1, pp. 267-276). CAADRIA. 